\title{
Ionization equilibrium of hot hydrogen plasma
}

\author{
Alexander Y. Potekhint \\ Department of Theoretical Astrophysics, Ioffe Physical-Technical Institute, 194021, St.-Petersburg, Russia
}

(Received 19 April 1996)

\begin{abstract}
The hydrogen plasma is studied at temperatures $T \sim$ $10^{4}-10^{6} \mathrm{~K}$ using the free energy minimization method. A simple analytic free energy model is proposed which is accurate at densities $\rho \lesssim 1 \mathrm{~g} \mathrm{~cm}^{-3}$ and yields convergent internal partition function of atoms. The occupation probability formalism is modified for solving the ionization equilibrium problem. The ionization degree and equation of state are calculated and compared with the results of other models.
\end{abstract}

PACS numbers: 95.30.Q, 95.30.T, 52.25.K

\section{INTRODUCTION}

Thermodynamic properties of astrophysical plasmas have been studied extensively in recent years [i]. The theoretical models are based either on the physical picture or on the chemical picture of the plasma [2]. In this paper we consider the latter. Our study is motivated by considerable disagreement among the existing models in the domain of partial ionization.

We consider the simplest case of pure hydrogen plasma which shows in relief all specific features of the problem. This particular case is also important for calculating atmospheric opacities of degenerate stars. It is generally assumed that, due to the gravitational stratification [3], outer spectra-forming layers of atmospheres of these stars consist of light elements. Observations [4] confirm that DA white dwarfs have virtually pure hydrogen atmospheres at temperatures up to $40000 \mathrm{~K}$.

It is worthwhile to mention two widely used theories for the hydrogen plasma. The first one has been elaborated by Hummer and Mihalas [5] (HM), and Mihalas, Däppen, and Hummer [6.7] (MDH). The authors presented a detailed discussion of previous work and formulated an equation of state (EOS), using an occupation probability formalism to obtain a finite internal partition function (IPF) of atoms. The occupation probabilities were derived from the plasma microfield distribution. The second theory has been proposed by Saumon and Chabrier 8 10] (SC) (see also Ref. [11]). These authors have developed a free energy model based on effective pair potentials in the system of hydrogen atoms, molecules, protons, and electrons. The theory describes successfully either a completely ionized plasma [8] or neutral gas [9] but suffers from difficulties in treating the partial ionization. The free energy models originally adopted by HM [5] and SC [9] did not ensure reasonable convergence of IPF at high temperature and pressure ionization at high density. This compelled the authors to introduce ad hoc modifications [6, 10] which affected the ionization equilibrium. The ionization curves obtained in Refs. [6] and 10] at $T>10^{4} \mathrm{~K}$ are strikingly different.

In this paper we study hydrogen plasma at $T \sim$ $10^{4}-10^{6} \mathrm{~K}$ when a considerable fraction of atoms can exist in excited states, and the IPF convergence becomes crucial. In Sect. II we outline the free energy minimization method. In Sect. III we develop an analytic free energy model for weakly coupled and weakly degenerate plasma. In Sect. IV] we revise the occupation probability formalism, and in Sect. V we calculate the EOS and ionization equilibrium, making a comparison with the results of other models.

\section{CHEMICAL PICTURE AND FREE ENERGY MINIMIZATION METHOD}

In the chemical picture of plasmas, bound objects (atoms, molecules, ions) are treated as elementary members of the thermodynamic ensemble, along with free electrons and nuclei. In the physical picture, nuclei and electrons (free and bound) are the only constituents of the ensemble.

Both pictures can be thermodynamically self-consistent, but the chemical picture has limited microscopic consistency. For instance, it does not provide a proper treatment of such cluster configurations as "an atom + a close alien ion" (a pseudomolecular ion). With increasing density, the ionization of an electron bound to a particular nucleus proceeds through a progressive delocalization involving cluster ("hopping") states [12]. These states are negligible in nearly ideal gases, but important at higher densities. However, their inclusion as new members of the thermodynamic ensemble would complicate the free energy model. Therefore one usually considers basic chemical species which dominate at low density.

On the other hand, the physical picture is commonly based on diagrammatic expansions which converge only at low densities. Thus one has to resort to additional assumptions in the frames of either picture, in order to progress on higher density. The chemical picture, combined with the free energy minimization method, represents a reasonable compromise between the rigorous treatment and the practical application.

The central assumption of the free energy minimization method [13] is the factorization of the many-body partition function into translational, configurational, and internal factors, and corresponding separation of the 
Helmholtz free energy $F$ :

$$
F\left(V, T,\left\{N_{\alpha}\right\}\right)=F_{\text {trans }}+F_{\text {conf }}+F_{\text {int }} .
$$

Here $V$ is the volume, $T$ the temperature, and $\left\{N_{\alpha}\right\}$ the set of particle numbers. The internal structure of a composite particle is generally affected by the surrounding, hence the separation (1) is approximate.

At given $V$ and $T$, the equilibrium state is determined by minimizing $F$ with respect to the numbers $\left\{N_{\alpha}\right\}$, subject to the stoichiometric constraints. Then the pressure $P$, the entropy $S$ and related quantities are obtained from $F$ using the well known thermodynamic relations [14.

\section{FREE ENERGY MODEL}

\section{A. Plasma parameters}

Consider a plasma of electrons, protons, and hydrogen atoms. We do not include molecules and molecular ions, assuming that the temperature is high enough for their dissociation (roughly, $T>10^{4} \mathrm{~K}$; possible departure from this assumption will be discussed in Sect.VD.

The charged component of the plasma is described by the coupling parameter $\Gamma$ and degeneracy parameter $\theta$,

$$
\Gamma=\beta e^{2} / a_{\mathrm{e}}, \quad \theta=T / T_{\mathrm{F}},
$$

where $\beta=\left(k_{\mathrm{B}} T\right)^{-1}, k_{\mathrm{B}}$ is the Boltzmann constant, $a_{\mathrm{e}}=\left(\frac{4}{3} \pi n_{\mathrm{e}}\right)^{-1 / 3}$ is the mean inter-electron distance, $n_{\mathrm{e}}=N_{\mathrm{e}} / V$ is the electron number density, and $T_{\mathrm{F}}$ is the Fermi temperature $\left(T_{\mathrm{F}} \approx 912 \mathrm{~K} n_{20}^{2 / 3}\right.$ and $\Gamma \approx$ $[12500 \mathrm{~K} / T] n_{20}^{1 / 3}$, where $\left.n_{20} \equiv n_{\mathrm{e}} / 10^{20} \mathrm{~cm}^{-3}\right)$. We consider weakly coupled and weakly degenerate plasma. The generalization to higher degeneracy $(\theta<1)$ is straightforward [14. The strong Coulomb coupling $(\Gamma>1)$ can be taken into account using the models developed in Refs. 8.,15.

\section{B. Translational free energy}

The free energy of the ideal plasma $F_{\text {id }}=F_{\text {trans }}+F_{\text {int }}$ is the sum of three terms produced by electrons (e), protons (p), and $\mathrm{H}$ atoms. For the atoms and protons,

$$
\begin{aligned}
& \beta F_{\mathrm{id}}^{(\mathrm{p})} / N_{\mathrm{p}}=\beta F_{\text {trans }}^{(\mathrm{p})} / N_{\mathrm{p}}=\ln \left(n_{\mathrm{p}} \lambda_{\mathrm{p}}^{3}\right)-1, \\
& \beta F_{\text {trans }}^{(\mathrm{H})} / N_{\mathrm{H}}=\ln \left(n_{\mathrm{H}} \lambda_{\mathrm{H}}^{3}\right)-1,
\end{aligned}
$$

where $n_{\alpha}=N_{\alpha} / V$ is the number density of species $\alpha$, and $\lambda_{\alpha}=\left(2 \pi \beta \hbar^{2} / m_{\alpha}\right)^{1 / 2}$ is the thermal de Broglie wavelength. We neglect the proton spin weight since it would yield an insignificant constant in the free energy. For the electrons, we include the spin weight and the low-density correction for the degeneracy [14]:

$$
\beta F_{\mathrm{id}}^{(\mathrm{e})} / N_{\mathrm{e}}=\ln \left(n_{\mathrm{e}} \lambda_{\mathrm{e}}^{3} / 2\right)-1+n_{\mathrm{e}} \lambda_{\mathrm{e}}^{3} / 2^{7 / 2} .
$$

\section{Internal free energy}

There are different ways to define the internal free energy of atoms $F_{\text {int }}^{(\mathrm{H})}$. We calculate it in the ideal gas approximation, neglecting interactions of atoms with surrounding particles. From the first principles [14], the ideal-gas part of the free energy is

$$
\beta F_{\mathrm{id}}^{(\mathrm{H})}=\sum_{\kappa} N_{\kappa}\left[\ln \left(n_{\kappa} \lambda_{\mathrm{H}}^{3} / g_{\kappa}\right)-1-\beta \chi_{\kappa}\right],
$$

where $\kappa$ enumerates quantum states with statistical weights $g_{\kappa}$ and non-perturbed binding energies $\chi_{\kappa}$. It has been shown [16] that the binding energies of an atom in a plasma practically do not shift with increasing density until they merge into the continuum. Comparing Eqs. (6) and (4), we obtain

$$
\beta F_{\text {int }}^{(\mathrm{H})}=\sum_{\kappa} N_{\kappa} \ln \left[N_{\kappa} /\left(N_{\mathrm{H}} g_{\kappa} \mathrm{e}^{\beta \chi_{\kappa}}\right)\right]
$$

Now all nonideality effects should be included in the configurational term $F_{\text {conf }}$. However, $F_{\text {conf }}$ does affect the equilibrium value of $F_{\mathrm{int}}^{(\mathrm{H})}$ through the distribution of the occupation numbers $\left\{N_{\kappa}\right\}$, which is not assumed to obey the ideal-gas Boltzmann law.

\section{Configurational free energy}

It is the common practice to separate Coulomb interactions of charged particles from interactions involving neutral atoms and to describe the first ones by the free energy of a pure Coulomb plasma. At low density, the excess free energy of the charged component is given by the Debye-Hückel theory with a two-component plasma quantum correction [17]:

$$
\beta F_{\mathrm{C}}=-\frac{2 e^{3}}{3} \sqrt{\frac{\pi \beta^{3}}{V}}\left(N_{\mathrm{e}}+N_{\mathrm{p}}\right)^{3 / 2}\left(1-\frac{3 \sqrt{\pi}}{2^{7 / 2}} \gamma\right)
$$

where $\gamma=\frac{1}{4}\left(\gamma_{\mathrm{ee}}+2 \gamma_{\mathrm{ep}}\right), \gamma_{\mathrm{ee}}$ and $\gamma_{\mathrm{ep}}$ being the electronelectron and electron-proton quantum diffraction parameters, $\gamma_{\mathrm{ep}} \approx \gamma_{\mathrm{ee}} / \sqrt{2}=2 \hbar \beta e \sqrt{\pi\left(n_{\mathrm{e}}+n_{\mathrm{p}}\right) / m_{\mathrm{e}}}$. Our $\gamma$ differs from the one-component plasma parameter $\gamma_{\mathrm{e}}$ employed in the SC model [8] by multiplier 0.854 .

The quantum corrections in Eqs. (5) and (8) allow us to extend the analytic free energy model from the low density region of $\Gamma \ll 1$ and $\theta \gg 1$ to the moderate density, where $\Gamma \lesssim 1, \theta \gtrsim 1$. We have checked (see also Fig. 12 of Ref. 8) that the account of these corrections extends the validity range of the model by more than an order of magnitude toward higher density or lower temperature.

The neutral component produces additional configurational terms, which describe interactions of neutral species with neutral and charged particles. For atoms 
and ions, these interactions are described by pair potentials, while the electrons are assumed to adjust to any configuration of the heavy particles. A free energy model based on this approach has been elaborated by SC [9, 10 and extended recently to helium plasma [18]. Our modified version of the pair potential free energy model $F_{\text {pair }}$ is given in Appendix. However, the contribution to the free energy, which comes from unbounding of atoms in the course of their interactions with other particles, is not fully griped at this approach. Namely, the effective hard sphere diameters of atoms, derived from effective binary potentials, appear to be too small to enforce ionization in high- $T$ and high- $\rho$ domain. Below we consider the problem in more detail.

\section{E. Pressure destruction of atoms}

The central problem of calculating the occupation numbers $\left\{N_{\alpha}\right\}$ is to achieve the self-consistent convergence of IPF, that is most difficult in the case of partial ionization. There have been many attempts to solve the problem; some of them are discussed in Ref. [5].

Clearly, a bound state cannot be populated, if the corresponding "size" of the electronic "orbit" (the electron cloud) is too large. In other words, the electron cannot be bound if there is not enough free space, or if it suffers from strong perturbations. The problem is how to include the unbounding into the chemical picture in a self-consistent manner.

The simplest way to obtain the convergence is to truncate the IPF at an appropriate "critical" quantum number $\kappa^{*}$, for example, corresponding to a quantummechanical size of an atom $l_{\kappa^{*}}$ comparable with the mean interparticle distance [19]. However, since $\kappa^{*}$ depends on the physical conditions (particularly, on density), the abrupt cutoff produces unrealistic discontinuities in the free energy. Continuous truncation procedures imply introduction of occupation probabilities $w_{\kappa}$ into the IPF, which suppress higher states and ensure the convergence. However, as has been shown by Fermi 20] and emphasized by HM [5], the introduction of $w_{\kappa}$ requires a modification of the free energy. Therefore $w_{\kappa}$ should be consistent with the adopted form of $F_{\text {conf }}$.

In the free energy model $F_{\text {pair }}$ (Appendix), the repulsive interparticle interactions are simulated by repulsion of hard spheres (HS) with appropriate diameters $d$. However, for a high $T$ or for a high atomic level, $d$ becomes much smaller than the atomic size $l_{\kappa}$, so that other plasma particles can penetrate the atom. If this happens, then the electronic orbit gets embedded into the plasma, which screens the attraction to the nucleus and makes the electron unbound. Then the atomic constituents should be extracted from the neutral component and treated as independent participants in the charged component. Similar situation (known as pressure ionization) occurs even for ground state atoms, if the density is high enough.
To allow for the plasma screening of the intra-atomic Coulomb interactions, the static screened Coulomb potential (SSCP) has been widely used (e.g., Ref. [21]). However this approach has been strongly criticized [5]. More consistent is the dynamical screening recently investigated in the framework of the thermodynamic Green function method 16. However, the practical need (e.g., in astrophysics) for large arrays of thermodynamic data necessitates looking for a simplified approach.

A convenient model has been described by HM. The configurational term given by Eq. (4.72) of Ref. [5] for the hydrogen plasma may be represented as

$$
F_{\mathrm{conf}}^{(\mathrm{HM})}=k_{\mathrm{B}} T \sum_{\kappa} N_{\kappa}\left(n_{\mathrm{H}} \tilde{v}_{1 \kappa}+n_{\mathrm{p}} \tilde{v}_{\mathrm{p} \kappa}\right)
$$

where $\tilde{v}_{1 \kappa}$ and $\tilde{v}_{\mathrm{p} \kappa}$ are characteristic volumes associated, respectively, with the atom-atom and atom-ion interactions. The first term includes the interaction of an atom in a state $\kappa$ with the ground state atom only. This is the "low excitation approximation" proposed by HM to make $F_{\text {conf }}$ linear in $N_{\kappa}$, which was essentially employed in deriving the occupation probabilities (despite the fact that even at this approximation the linearity obviously breaks down for the ground state atoms). For purely neutral gas $\left(n_{\mathrm{p}}=0\right)$, Eq. (9) reproduces the free energy derived by Fermi [20]. The atom-ion interaction volume $\tilde{v}_{\mathrm{p} \kappa}$ in this approach is due to microfield perturbations; it depends on the principal quantum number in a complicated way, but it is always $1-2$ orders of magnitude larger than $\tilde{v}_{1 \kappa}$. For example, for the ground-state hydrogen, Eq. (4.70) of Ref. [5] yields $\tilde{v}_{\mathrm{p} 1}=128(4 \pi / 3) a_{\mathrm{B}}^{3}$, where $a_{\mathrm{B}}$ is the Bohr radius.

It was found [6] that this model does not provide pressure ionization at high density, which is not surprising. Indeed, since $\tilde{v}_{\mathrm{p} \kappa} \gg \tilde{v}_{1 \kappa}, F_{\text {conf }}^{(\mathrm{HM})}$ may increase more rapidly with growing $n_{\mathrm{p}}$ than with $n_{\mathrm{H}}$, shifting the equilibrium towards lower ionization degree at higher densities. In order to ensure the desired ionization, MDH introduced an artificial "pressure ionization term" $F_{5}$ into the free energy, which rapidly increased whenever the density of neutral fraction exceeded $10^{-2} \mathrm{~g} \mathrm{~cm}^{-3}$. It can be shown that it is this term (and not the inaccurate HS treatment, as supposed in Ref. [11]) that produces an unrealistically stiff EOS at $\rho>10^{-2} \mathrm{~g} \mathrm{~cm}^{-3}$.

As argued in Refs. [19,20, 5], the quantum-mechanical atomic size $l_{\kappa}$ [Eq. (A9)] should not exceed the mean interparticle distance. This is not a problem at low temperature, when the effective HS diameters $d_{\kappa \kappa^{\prime}}$ are larger than $l_{\kappa}$. At high $T$, however, $d_{\kappa \kappa^{\prime}}$ become small, allowing configurations with strongly overlapping wavefunctions of neutral atoms. SC escaped this difficulty by introducing an additional hard core [Eq. (14) of Ref. [10]] in the effective potentials. However, a large hard core inside the atom seems to be unrealistic. We propose a modification of the free energy, which has another interpretation.

An electron can be treated as bound to a particular nucleus, if only its wave function does not overlap 
strongly with wave functions of other electrons, either free or bound to neighboring atoms. From the classical point of view, the atomic electron becomes unbound when another electron falls inside its orbit and shields the attraction to the nucleus. This resembles the plasma screening of the nucleus in the SSCP model but does not imply the collective nature of the screening.

For a given state $\kappa$, the probability that such unbounding does not occur can be estimated at low density from the Poisson distribution, $p_{\kappa}=\exp \left[-n_{\mathrm{c}} v_{\kappa}\right]$, where $n_{\mathrm{c}}=\left(n_{\mathrm{e}}+n_{\mathrm{H}}\right)$ is the total number of randomly distributed electronic clouds (including those which are bound to nuclei), and $v_{\kappa}=\frac{4}{3} \pi l_{\kappa}^{3}$. The unbounding requires to exclude the overlapping configurations from the total partition function, thus reducing the volume of the phase space available to the system. Equivalently, the existence of an atom in the state $\kappa$ corresponds to an event with probability $p_{\kappa}$ and thus diminishes the entropy. The total negentropy corresponding to a set of occupation numbers $\left\{N_{\kappa}\right\}$ is $-\sum_{\kappa} N_{\kappa} \ln p_{\kappa}$, which gives the free energy contribution

$$
F_{\mathrm{ub}}=k_{\mathrm{B}} T N_{\mathrm{c}} n_{\mathrm{H}} \bar{v}_{\mathrm{H}}
$$

where $\bar{v}_{\mathrm{H}}=\sum_{\kappa} N_{\kappa} v_{\kappa} / N_{\mathrm{H}}$ is the average atomic volume.

A similar term has been introduced by HM who, however, considered the destruction of atoms by microfields fluctuating due to the motions of surrounding heavy particles (as discussed in Sect. IVb(ii) of Ref. [5]). Although we readily agree that nearby passages of positive ions can ionize a particular atom, this process does not affect the occupation numbers at the thermodynamic equilibrium, since it is compensated by the inverse (neglected) process, owing to the principle of detailed balance. The net effect of both processes is not given in advance but itself should be determined from the thermodynamic equilibrium conditions. In contrast, the unbounding by an excessive negative charge occurring inside an electron orbit has no balancing counterpart. Moreover, it seems inconsistent to include any dynamical process dependent on particle momenta into $F_{\text {conf }}$ after separating the translational term $F_{\text {trans }}$, because the separation (11) implies that the other terms may depend only on particle configuration coordinates. For this reason we do not consider atomic collisions with ions [5] and free electrons [22].

Additional arguments in favor of the modification (10) of the excess free energy (9) will be given in Sect. IVB.

Finally, the total free energy

$$
F=F_{\text {id }}+F_{\mathrm{C}}+F_{\text {pair }}+F_{\text {ub }}
$$

is given by Eqs. (3), (5), (6), (8), (10), and (A21).

Other chemical species can be easily included in our analytic model. For example, $\mathrm{H}_{2}$ molecules can be taken into account by adding van der Waals and HS terms with appropriate effective diameters in Eqs. (A11), (A16) and (A19). The generalization of the unbounding term is

$$
F_{\mathrm{ub}}=k_{\mathrm{B}} T N_{\mathrm{c}}\left(n_{\mathrm{H}} \bar{v}_{\mathrm{H}}+n_{\mathrm{H} 2} \bar{v}_{\mathrm{H} 2}\right),
$$

where $n_{\mathrm{c}}=n_{\mathrm{e}}+n_{\mathrm{H}}+n_{\mathrm{H} 2}$, and $\bar{v}_{\mathrm{H} 2}$ is the average molecular volume.

\section{OCCUPATION PROBABILITY FORMALISM}

\section{A. Generalized Saha equation}

The internal free energy (7) can be rewritten in a more familiar form. Let us replace the Boltzmann distribution $N_{\kappa} \propto g_{\kappa} \exp \left(\beta \chi_{\kappa}\right)$ (which yields the divergent ideal IPF) by any real distribution

$$
N_{\kappa}=N_{\mathrm{H}} w_{\kappa} g_{\kappa} \exp \left(\beta \chi_{\kappa}\right) / Z_{w}
$$

where the generalized IPF

$$
Z_{w}=\sum_{\kappa} g_{\kappa} w_{\kappa} \mathrm{e}^{\beta \chi_{\kappa}}
$$

plays role of a normalization constant. Then

$$
\beta F_{\mathrm{int}}^{(\mathrm{H})}=\sum_{\kappa} N_{\kappa} \ln w_{\kappa}-N_{\mathrm{H}} \ln Z_{w} .
$$

Note that $w_{\kappa}$ and $Z_{w}$ in Eqs. 13)-(15) can be multiplied by a common factor. It does not affect the $N_{\kappa} / N_{\mathrm{H}}$ distribution, but should be chosen consistent with the ionization equilibrium conditions.

Although the first sum in Eq. (15) is naturally derived from the rigorous Eq. (6), it was often omitted in the internal free energy [9] or regarded as a part of $F_{\text {conf }}$ [5]. Meanwhile, it has a clear physical meaning: $-N_{\kappa} \ln w_{\kappa}$ is a contribution to the ideal-gas part of the entropy due to the correction $w_{\kappa}$ to the probability that $\kappa$ th state is occupied. The factors $w_{\kappa}$ are traditionally called occupation probabilities, although they do not always have direct probability meaning.

The minimum of the Helmholtz free energy under the stoichiometric constraints requires

$$
\partial F / \partial N_{\kappa}=\partial F / \partial N_{\mathrm{p}}+\partial F / \partial N_{\mathrm{e}}
$$

Separation of $F_{\text {id }}$ from $F_{\text {conf }}$ allows one to rewrite Eq. (16) in the form of the Saha equation:

$$
n_{\kappa}=n_{\mathrm{p}} n_{\mathrm{e}}\left(\lambda_{\mathrm{p}} \lambda_{\mathrm{e}} / \lambda_{\mathrm{H}}\right)^{3} w_{\kappa}\left(g_{\kappa} / 2\right) \exp \left[\beta\left(\chi_{\kappa}+\Lambda_{\mathrm{deg}}\right)\right],
$$

where $\Lambda_{\text {deg }}=n_{\mathrm{e}} \lambda_{\mathrm{e}}^{3} / 2^{5 / 2}$ is the correction due to the partial electron degeneracy, and $w_{\kappa}$ is defined by

$$
k_{\mathrm{B}} T \ln w_{\kappa}=\frac{\partial F_{\mathrm{conf}}}{\partial N_{\mathrm{p}}}+\frac{\partial F_{\mathrm{conf}}}{\partial N_{\mathrm{e}}}-\frac{\partial F_{\mathrm{conf}}}{\partial N_{\kappa}} .
$$

This definition is consistent with Eqs. (13)-(15), and it fixes the above mentioned common factor. These occupation probabilities have the same meaning as those considered by HM but take into account charged particles. Therefore, Eq. (18) generalizes Eq. (2.18) of Ref. [5] 
to the case when ionization-recombination processes are allowed in the system.

Equivalently, one may adhere the traditional definition, $\ln w_{\alpha}=-\beta \partial F_{\text {conf }} / \partial N_{\alpha}$, and replace $w_{\kappa}$ in Eq. (17) by $w_{\kappa} /\left(w_{\mathrm{e}} w_{\mathrm{p}}\right)$. However, since proton and electron cannot be destroyed by the external fields, we put their "occupation probabilities" equal to unity, thus choosing the definition (18) for the atomic occupation factors $w_{\kappa}$.

An equation equivalent to Eqs. (17) and (18) was derived by Fontaine et al. [21] (FGVH), who however did not introduce the occupation probabilities explicitly.

If all $w_{\kappa}$ were known, then Eq. (17) would give direct solution to the problem. However, since $F_{\text {conf }}$ depends on the occupation numbers, Eqs. (17) and (18) are to be solved together. Nevertheless, this reformulation of the problem is useful, because the coupled equations can be solved iteratively. First, one chooses an initial value of $w_{\kappa}$ and calculates the particle numbers $\left\{N_{\alpha}\right\}$ from Eq. (17). Then $w_{\kappa}$ are refined by substituting $\left\{N_{\alpha}\right\}$ into Eq. (18). At low densities, where many excited states are populated, this procedure appears to be more efficient than alternative schemes [6,13,21.

Substituting Eqs. (11) and (A21) into Eq. (18) we decompose $w_{\kappa}$ into five factors,

$$
w_{\kappa}=w_{\kappa}^{(\mathrm{ub})} w^{(\mathrm{C})} w_{\kappa}^{(\mathrm{HS})} w_{\kappa}^{(\mathrm{H})} w_{\kappa}^{(\mathrm{in})},
$$

corresponding to the unbounding of atoms, the Coulomb interactions of charged particles, the hard-sphere repulsion, and the corrections due to atom-atom and atom-ion attraction. Equation (10) yields

$$
w_{\kappa}^{(\mathrm{ub})}=\exp \left[-n_{\mathrm{c}} v_{\kappa}\right]
$$

Expanding $F_{\mathrm{C}}$ and $F_{\text {pert }}$ in powers of particle numbers, keeping quadratic terms, and using Eq. (18), we obtain

$$
\begin{aligned}
& \ln w^{(\mathrm{C})}=-\sqrt{4 \pi\left(\beta e^{2}\right)^{3}\left(n_{\mathrm{e}}+n_{\mathrm{p}}\right)}(1-\sqrt{\pi / 8} \gamma), \\
& \ln w_{\kappa}^{(\mathrm{H})}=2 \beta \sum_{\kappa^{\prime}} n_{\kappa^{\prime}} a_{\kappa \kappa^{\prime}}, \\
& \ln w_{\kappa}^{(\mathrm{in})}=\beta n_{\mathrm{p}} a_{\kappa}-\beta \sum_{\kappa^{\prime}} n_{\kappa^{\prime}} a_{\kappa^{\prime}},
\end{aligned}
$$

where $a_{\kappa \kappa^{\prime}}$ and $a_{\kappa}$ are the van der Waals constants defined in Appendix. Our calculations show that the factors $w^{(\mathrm{in})}$ and $w^{(\mathrm{H})}$ are close to unity, being, thus, unimportant. In contrast, $w^{(\mathrm{C})}$ is significantly less than unity at high densities, even at a relatively low ionization.

For the HS repulsion, Eq. (A20) gives

$$
\ln w_{\kappa}^{(\mathrm{HS})}=\frac{\left(\ln w_{\kappa}^{(0)}\right)(1-\eta / 2)-5 \eta^{2}+3 \eta^{3}}{(1-\eta)^{3}},
$$

where $\eta$ is the filling factor, and

$$
\ln w_{\kappa}^{(0)}=-\frac{4 \pi}{3}\left[\sum_{\kappa^{\prime}} n_{\kappa^{\prime}}\left(d_{\kappa \kappa^{\prime}}^{3}-d_{\kappa^{\prime}}^{3}\right)+n_{\mathrm{p}} d_{\kappa}^{3}\right] .
$$

If $\eta \ll 1$, then $w^{(\mathrm{HS})} \approx w^{(0)}$. In practice, the non-linear corrections given by Eq. (24) may be important. We always have $w^{(\mathrm{HS})}<1$, since $d_{\kappa^{\prime}}<d_{\kappa \kappa^{\prime}}$ in our model.

The formalism can be generalized, e.g., for formation of molecules. The dissociation-recombination equilibrium is given by

$$
n_{\mathrm{H} 2}=n_{\mathrm{H}}^{2}\left(\lambda_{\mathrm{H}} \sqrt{2}\right)^{3} Z_{w 2} / Z_{w}^{2}
$$

where $Z_{w 2}$ is the internal molecular partition function, generalized through multiplying each $\mu$ th term by an occupation probability $w_{\mu}^{(\mathrm{H} 2)}$ (e.g., Eq. (21) of Ref. [9]). From the equation of chemical equilibrium

$$
\partial F / \partial N_{\mathrm{H} 2}=2\left(\partial F / \partial N_{\mathrm{p}}+\partial F / \partial N_{\mathrm{e}}\right)
$$

we conclude that the molecular occupation probability can be defined as

$$
k_{\mathrm{B}} T \ln w^{(\mathrm{H} 2)}=2\left(\frac{\partial F_{\mathrm{conf}}}{\partial N_{\mathrm{p}}}+\frac{\partial F_{\mathrm{conf}}}{\partial N_{\mathrm{e}}}\right)-\frac{\partial F_{\mathrm{conf}}}{\partial N_{\mathrm{H} 2}} .
$$

Keeping the traditional definition of $w$ both for atoms and for molecules would result in the same Eq. (26).

For the perturbation and HS factors in $w^{(\mathrm{H} 2)}$, Eqs. (22)-(25) remain valid if to use relevant scaling factors; the Coulomb factor $w^{(\mathrm{C})}$ should be squared; and the unbounding factor is derived from Eq. (12):

$$
\ln w_{\mu}^{(\mathrm{H} 2, \mathrm{ub})}=-n_{\mathrm{H}}\left(v_{\mu}-\bar{v}_{\mathrm{H}}\right)-n_{\mathrm{H} 2}\left(v_{\mu}-\bar{v}_{\mathrm{H} 2}\right)-n_{\mathrm{e}} v_{\mu} .
$$

As a test example (although marginal to the present discussion), we have implemented this approach to molecular formation, utilizing a simplified treatment of $Z_{w 2}$ 23,24. In this case, the unbounding factor (29) turned out to be unimportant, since the degree of dissociation is mainly determined by relation between atomic and molecular effective HS diameters.

The situation is very different for the ionization of atoms. The repulsion factor $w^{(\mathrm{HS})}$ becomes important at moderate $\rho$ and low $T$, but $w^{(\mathrm{ub})}$ strongly affects mostly excited states at any density (the ground state at high density), decreasing rapidly as the conditions for state survival become violated. This leads to a physically reasonable convergence of the IPF at low densities (thus radically improving the ionization degree at $T \gtrsim 10^{4.5} \mathrm{~K}$ ) and pressure ionization at high densities.

\section{B. Thermodynamic and optical occupation probabilities}

The occupation probability technique described above allows one to calculate thermodynamic properties of partially ionized hydrogen plasma. However, it is still insufficient for describing optical properties of the plasma.

The free energy model presented in Sect. III and in Appendix allows for close configurations of atoms with 
protons, because the atom-ion repulsion diameters $d_{\kappa}$ do not exceed the quantum-mechanical sizes of atoms. As argued in Sect. II, the close configurations simulate cluster states (approximately treated as interacting atoms and ions in the frames of the chemical picture). This approach yields physically plausible EOS. On the other hand, specific quantum-mechanical properties (e.g., frequencies and oscillator strengths of radiative transitions) of clusters most likely differ from those of isolated atoms. Formation of many different close configurations should manifest itself in optics as quasicontinuum.

Therefore, one should discriminate between the thermodynamic continuum (the states which do not contribute to the generalized IPF), and the optical continuum (the states strongly perturbed by surrounding). This dichotomy was first realized by Rogers [25], who developed the concept of the optical and plasma continua using the physical picture. A correct account of the quasicontinuum has been also taken in a recent study of line shapes in hydrogen opacities [26].

The optical continuum can be determined from consideration of Stark merging of spectral lines of an atom affected by plasma microfields [27. This leads to the atomic "survival" probabilities $\tilde{w}_{\kappa}$, generally different from $w_{\kappa}$ introduced in thermodynamics. Let us call $\tilde{w}_{\kappa}$ the optical occupation probability, to avoid confusion with $w_{\kappa}$. The occupation probabilities based on the plasma microfield distribution [5] [7] are in fact the optical ones. Their implication in thermodynamics leads to physically unrealistic EOS (cf. Ref. 11]) due to the incorrect treatment of close configurations in the free energy. Ionization equilibrium would be equally implausible without an ad hoc "pressure ionization" term [6]. Indeed, substituting the excess free energy (9) into Eq. (16) we would arrive at Eq. (17) with $w_{\kappa}$ replaced by

$$
w_{\kappa}^{(\mathrm{HM})}=\exp \left[-n_{\mathrm{H}} \tilde{v}_{1 \kappa}-n_{\mathrm{p}} \tilde{v}_{\mathrm{p} \kappa}+\sum_{\kappa^{\prime}} n_{\kappa^{\prime}}\left(\tilde{v}_{\mathrm{p} \kappa^{\prime}}-\tilde{v}_{1 \kappa^{\prime}}\right)\right] .
$$

Since $\tilde{v}_{\mathrm{p} \kappa} \gg \tilde{v}_{1 \kappa}$, the last (positive) term in Eq. (30) may dominate and yield the "occupation probabilities" which grow exponentially with $n_{\mathrm{H}}$. Then one would get pressure neutralization instead of pressure ionization at high densities 28].

In contrast, Eq. (10) leads to the occupation probabilities, Eq. (20), which decrease exponentially with density and produce the desired pressure ionization.

The occupation probabilities given by Eq. (4.71) of Ref. [5] can be presented (for pure hydrogen plasma) in the form (30) but without the last term. This form of $w_{\kappa}$ cannot be rigorously derived from Eq. (9). Note, however, that the leading factor $n_{\mathrm{p}} \tilde{v}_{\mathrm{p} \kappa}$ in the exponent occurs due to the Inglis-Teller effect [27] which is optical but not thermodynamic. Accordingly, these results can be used for calculating the fraction of atoms which are only slightly perturbed by plasma microfields so that they are able to contribute to the atomic opacities. For this purpose, we use an expression similar to that in Ref. [5]. However we take into account that $F_{\text {pair }}$ includes the HS term, which implies that the distance between ions and atoms cannot be shorter than $d_{\kappa}$. Thus the HS volume should be subtracted from the interaction volume $\tilde{v}$. The latter one has been estimated by several authors using different (not always justified) approximations, as discussed by HM [5]. A reasonable order-of-magnitude estimate reads $\tilde{v}=\frac{4}{3} \pi\left(4 l_{\kappa}\right)^{3}$. For 7 lowest states this estimate is intermediate between more complicated Eqs. (4.69) and (4.70) of Ref. [5], and for the ground state atom it fits the latter exponential with an accuracy of $9 \%$. Finally, we adopt

$$
\tilde{w}_{\kappa}=\exp \left[-\frac{4 \pi}{3} n_{\mathrm{p}}\left(\left(4 l_{\kappa}\right)^{3}-d_{\kappa}^{3}(T)\right)\right] .
$$

The above considerations emphasize that $\tilde{w}_{\kappa}$ determine solely the optical properties of the plasma and they should not be used in the construction of the IPF.

\section{RESULTS AND DISCUSSION}

\section{A. Ionization equilibrium}

The free energy model described in Sect. III has been applied to calculation of the thermodynamic properties of plasma using the method of Sect. IV. The ionization isotherms are shown in Fig. 1. Light solid curves represent the fraction of all $\mathrm{H}$ atoms, $f_{\mathrm{H}}=n_{\mathrm{H}} /\left(n_{\mathrm{H}}+n_{\mathrm{p}}\right)$, and dashed lines display the fraction of ground state atoms. The results are in general agreement with Ref. [10 but disagree with Ref. [6]. If, for example, $\rho=0.1 \mathrm{~g}$ $\mathrm{cm}^{-3}$, Fig. 2 of Ref. [6] shows practically zero ionization at $T \leq 10^{4.5} \mathrm{~K}$, whereas according to Ref. [10] there is a considerable amount (about $6 \%$ by mass) of free protons at $T=22000 \mathrm{~K}$. Our result coincides with the latter one. The pressure ionization in our Fig. 1 proceeds smoothly at high densities, again in agreement with Ref. [10], but contrary to almost abrupt pressure ionization of Ref. [6].

Thick solid and dashed lines in Fig. 1 are obtained using Eq. (31) and show the fraction of those atoms whose optical properties are not destroyed by plasma microfields, and which therefore should be used in the opacity calculations. At $\rho \lesssim 10^{-3} \mathrm{~g} \mathrm{~cm}^{-3}$, these curves are in good agreement with those in Ref. [6]. This observation suggests a possible explanation to a discrepancy in occupation numbers of excited states, recently recognized 29] between OPAL and MDH data: the former ones take into account all thermodynamically significant while the latter ones only optically identifiable atomic states.

For comparison, long dashes show the solution of the ideal-gas Saha equation including the ground state atoms only. These curves reproduce accurately the number of ground state atoms at low densities. However, at 
$T \gtrsim 10^{4.5} \mathrm{~K}$ the total number density $n_{\mathrm{H}}$ can never be determined in this way, since the excited states become populated and increase the neutral fraction. Above $10^{5} \mathrm{~K}$, the onset of occupation of the excited states produces typical "shoulders" on the solid curves. Note that highest populated excited states at low densities are strongly affected by microfields and belong to the optical quasicontinuum. This explains why the low-density tails of the heavy lines lie significantly lower than the light ones. This difference is noticeable not only in the pressure ionization domain $\rho \gtrsim 0.1 \mathrm{~g} \mathrm{~cm}^{-3}$ (where the present model has a limited applicability), but also at lower densities, if the temperature is high enough for population of the excited states. On the other hand, when density increases, the pressure ionization comes into effect, the neutral fraction becomes smaller and finally disappears at $\rho \gtrsim 3 \mathrm{~g}$ $\mathrm{cm}^{-3}$. There is a considerable amount of bound species at $\rho \sim 0.1-1 \mathrm{~g} \mathrm{~cm}^{-3}$ (important for thermodynamics) which can hardly contribute to the atomic opacities. The optical properties of atoms are destroyed at $\rho>10^{-2} \mathrm{~g}$ $\mathrm{cm}^{-3}$, as is seen from downward bending of the heavy lines.

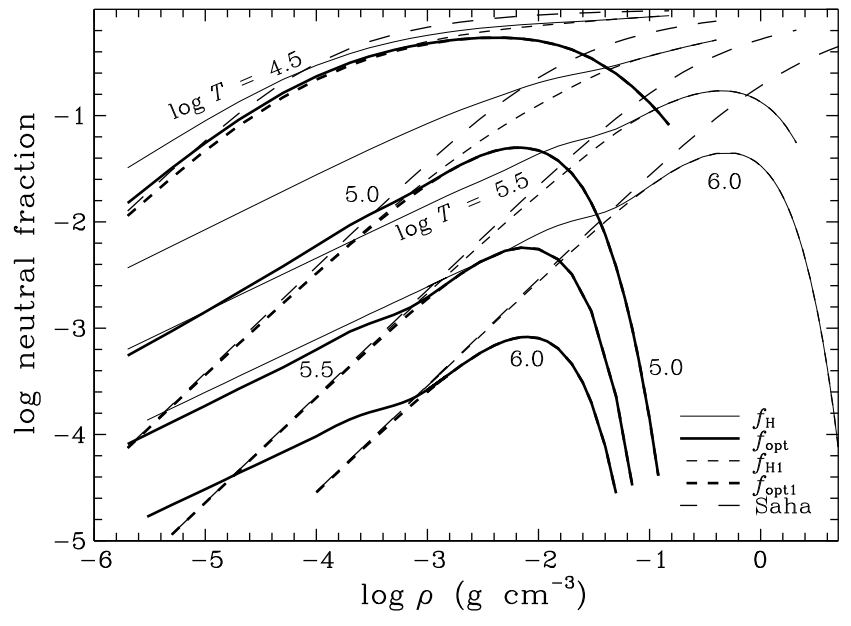

FIG. 1. Total neutral fraction $\left(f_{\mathrm{H}}\right)$ and partial fractions: ground state atoms $\left(f_{\mathrm{H} 1}\right)$, optically identifiable bound atoms $\left(f_{\text {opt }}\right)$, and optically identifiable ground state atoms $\left(f_{\text {opt } 1}\right)$, compared with the ideal gas (Saha) approximation.

\section{B. Equation of state}

Relative importance of partial contributions to the free energy can be estimated by examination of their influence on EOS, as illustrated in Fig. 2 for two temperatures. At lower temperature, $T=12600 \mathrm{~K}$, the ionization degree is small, and the corrections due to the Coulomb nonideality of the charged component are practically unimportant. The most important corrections are produced by the repulsion of atoms (HS) and by the unbounding. The corresponding contributions to the pressure are nearly equal and become appreciable at $\rho \gtrsim 0.03 \mathrm{~g} \mathrm{~cm}^{-3}$. At higher temperature, $T=2 \cdot 10^{5} \mathrm{~K}$, the ionization is high, and the Coulomb nonideality is significant, while the HS contribution is nearly negligible. The unbounding, however, is important at this temperature also. Note that the perturbation (van der Waals) terms are unimportant at any $T$ and $\rho$ shown in Fig. 2 .

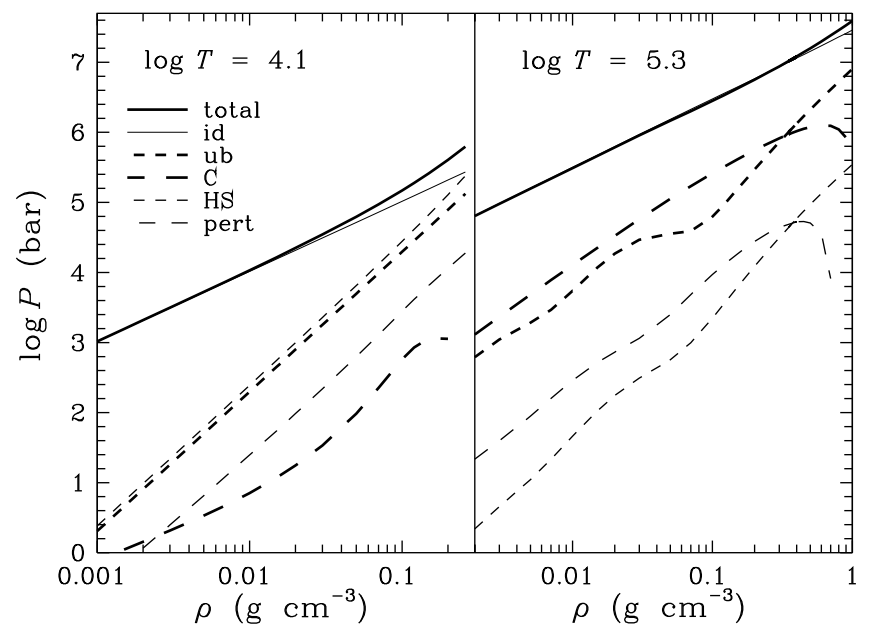

FIG. 2. Partial pressures: ideal gas (id) and configurational parts due to the Coulomb interactions of the ionized fraction (C), unbounding of atoms (ub), strong repulsion at short distances (HS), and long-distance attraction (pert). We show $\left|P_{\mathrm{C}}\right|$ and $\left|P_{\text {pert }}\right|$ since these parts are negative.

In Fig. 3 we compare the present EOS (heavy solid curves) with those known in the literature. Results of FGVH [21] are shown by dashes, MDH [6] by dot-dashed lines, SC [11 by light solid lines, and OPAL [30 by dots. The EOS of SC is somewhat softer, while that of $\mathrm{MDH}$ much stiffer (probably owing to the rapidly increasing "pressure ionization" term introduced by the latter authors). We have terminated our curves at densities where the quantum correction in Eq. (8) reached 0.5. At higher $\rho$ the nonideality of the charged component becomes too strong to be treated as perturbation. The lowest isotherm corresponds to $T=12600 \mathrm{~K}$, which is below the critical temperature for the plasma phase transition reported by SC [10] (the density discontinuity seen on the corresponding curve).

Our results have been obtained under the assumption that the molecules are completely destroyed. All authors agree that it is true at $T>10^{4.5} \mathrm{~K}$, however there is a great uncertainty concerning the degree of dissociation at $4.1 \lesssim \log _{10} T \lesssim 4.5$. According to Ref. [6], the amount of molecules in this interval is quite insignificant at any density, while SC [10,11] and Reinholz et al. 23] (RRN) found similarly strong but quantitatively different recombination at density increasing from $10^{-2}$ toward 1 $\mathrm{g} \mathrm{cm}^{-3}$. Quantum molecular dynamics simulations [31] show that there is a significant amount of transient $\mathrm{H}_{2}$ like clusters at $k_{\mathrm{B}} T=1 \mathrm{eV}$ and $\rho \sim 1 \mathrm{~g} \mathrm{~cm}^{-3}$, although their influence on thermodynamic and optical properties of plasma is not yet well understood. 


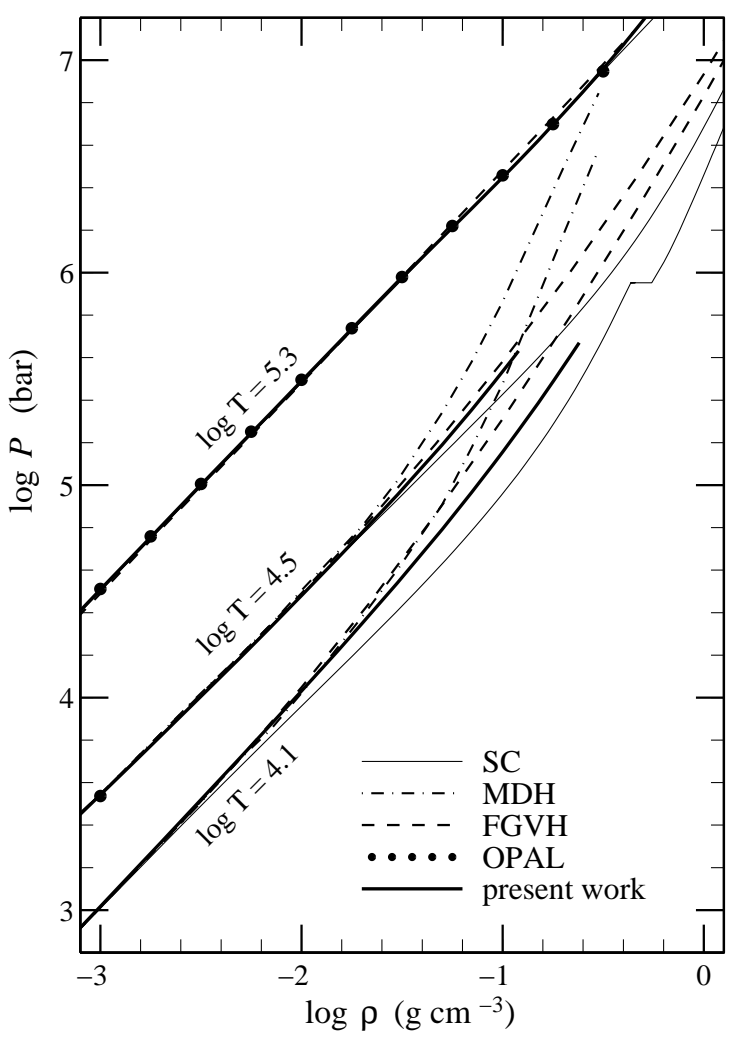

FIG. 3. Comparison of present EOS with results from the literature.

Figure 4 illustrates the effect of this uncertainty on the EOS. The pressure isotherm $(T=15000 \mathrm{~K})$ corresponding to complete dissociation (heavy solid line) is compared with two modified isotherms, obtained through replacing in our model the ideal contribution to $P$ by that corresponding to the dissociation degree given either by SC (dot-dashed line) or by RRN (dashed line). A comparison with the isotherm of SC (drawn by light solid line) suggests that the difference between our and SC EOS at $\log _{10} T \lesssim 4.5$ is mainly caused by the formation of dimers, which we neglected. Our testing calculations revealed, however, that the dissociation degree in the considered $\rho-T$ domain depends crucially on treatment of various molecular excitations as well as on adopted HS diameters. Thus the thorny problem of clustering hardly can get an unambiguous solution within the chemical picture.

Figure 5 shows the adiabatic temperature gradient $\nabla_{\text {ad }}=(\partial \ln T / \partial \ln P)_{S}$, a significant quantity sensitive to details of the free energy model. Thermal radiation starts to dominate in thermodynamics for the hottest isotherm at low density, causing the decrease of $\nabla_{\text {ad }}$. Other depressions of $\nabla_{\text {ad }}$ are explained by the increase of the specific heat in the regions of partial ionization, where the internal energy is affected by the strongly $T$-dependent ionization degree. Our data (heavy curves) are compared with the tables of FGVH 21 (dashes), SC 11 (dot-dashed lines, "table") and OPAL 30] (dots). Light solid lines ("formula") are obtained by substituting the SC tabulated quantities $P, S, T, a=(\partial \log \rho / \partial \log T)_{P}$ and $b=(\partial \log S / \partial \log T)_{P}$ into the thermodynamic identity $\nabla_{\mathrm{ad}}=-a P V /(b S T)$. We have plotted only the latter lines for the two highest isotherms, for which the "table" and "formula" values are in a good agreement. The discrepancy between these values in the low- $T$ high$\rho$ domain indicates the lack of thermodynamic consistency 11 caused by a high sensitivity of the complicated numerical approach to accidental small errors in minimization and differentiation procedures. Although our simplified analytic model is less scrupulous in details, its advantage is that it is free of such inconsistencies.

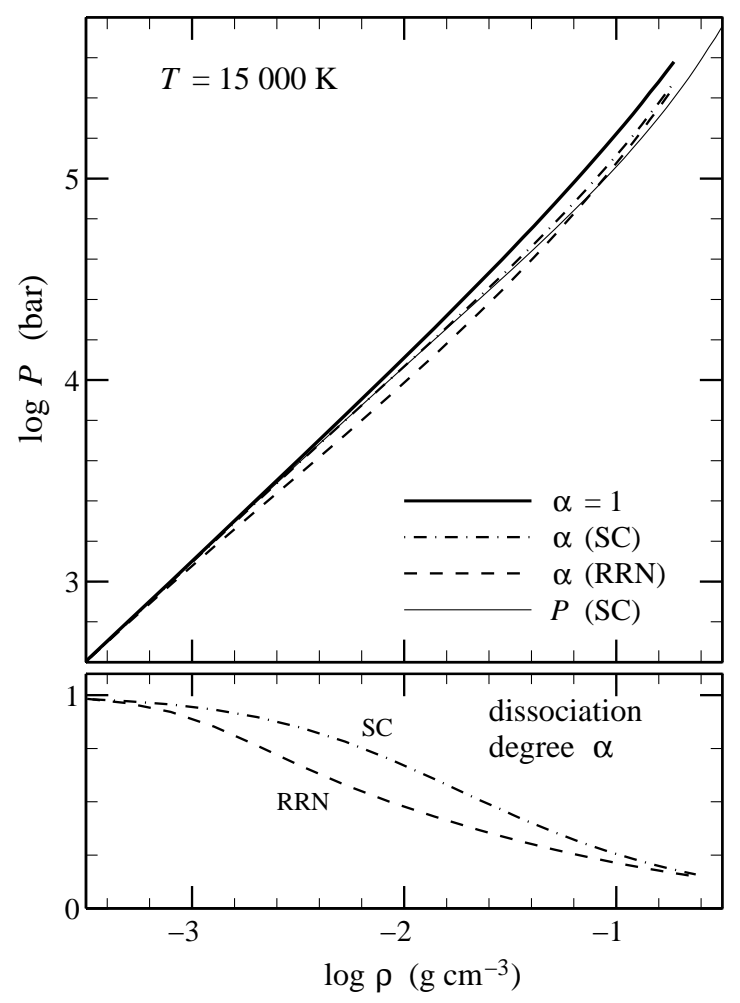

FIG. 4. Influence of the uncertainty in the dissociation degree at high density on the EOS.

The most crucial test for the validity of our model may be provided by a comparison with the advanced results based on the physical picture and employed in the OPAL opacity library [30]. They do not cover the most interesting region where other models reveal major discrepancies, but the available data are in satisfactory agreement with our model.

Sequences of shallow depressions in the isotherms $T \geq$ $10^{5} \mathrm{~K}$ at $\rho>10^{-2} \mathrm{~g} \mathrm{~cm}^{-3}$ indicate successive pressure destruction of excited atomic states. Their physical reality remains an open question. Note that the SC results reveal analogous oscillatory behavior (which is probably inherent to the models of such a type), which however is not observed in the OPAL data. 

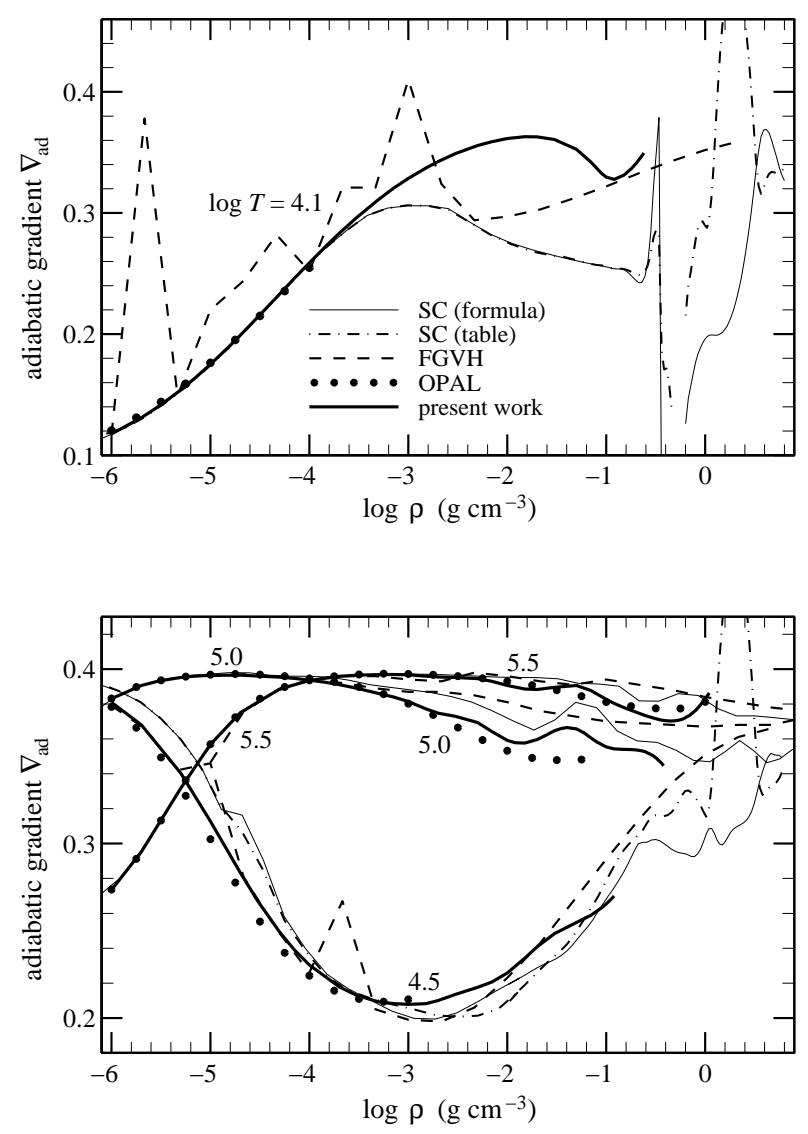

FIG. 5. Adiabatic temperature gradient compared with the data of FGVH, SC, and OPAL. The curves are labeled by $\log _{10} T(\mathrm{~K})$.

\section{SUMMARY}

We have developed an analytic free energy model for partially ionized hydrogen plasma in the framework of the chemical picture. The model describes thermodynamic properties of the plasma at $T>10^{4} \mathrm{~K}$ and $\rho \lesssim 0.1-1 \mathrm{~g} \mathrm{~cm}^{-3}$. In particular, it can be used in the studies of DA white dwarf and neutron star atmospheres.

The occupation probability formalism, first introduced by Fermi [20] and further developed by Hummer and Mihalas [5], is generalized to take proper account of the effects of partial ionization. Free energy minimization is obtained by a generalized Saha equation which is solved by an iterative algorithm involving the modified occupation probabilities. Calculated ionization degree differs from that obtained previously in Ref. [6], but qualitatively agrees with the results of Ref. [10]. We argue that the relatively high neutral fraction obtained in Ref. 10 and in our present work at $\rho \sim 0.1-1 \mathrm{~g} \mathrm{~cm}^{-3}$ cannot be pronounced in atomic opacities, since it takes cumulative account of atomic and cluster states, the latter ones contributing to the optical quasicontinuum (Sect. [IVB]). We introduce the optical occupation probabilities which determine the neutral fraction visible in atomic lines. The non-perturbed atomic fraction given by these probabilities agrees with that of Ref. [6] at the densities available in laboratory.

The equation of state obtained from our model is compared with the results of other authors. The best agreement is achieved with the OPAL data [30] (in the $\rho-T$ region where they are available). Since the equation of state employed in OPAL is based on the physical picture of plasma, completely different from our model, we regard this agreement as an indirect confirmation of the validity of our approach. Its generalization to higher densities and higher atomic numbers is being performed.

\section{ACKNOWLEDGMENTS}

I am pleased to acknowledge useful discussions with G. G. Pavlov, Y. A. Shibanov, and D. G. Yakovlev, valuable comments of G. Chabrier, F. J. Rogers, and D. Saumon, and consultations with V. E. Zavlin. The hospitality of Gilles Chabrier at the Ecole Normale Supérieure de Lyon is gratefully acknowledged. The work was supported in part by RBRF (Grant 96-02-16870) and INTAS (Grant 94-3834).

\section{APPENDIX: FREE ENERGY MODEL BASED ON INTERPARTICLE PAIR POTENTIALS}

Our model correction to the excess free energy $F_{\text {pair }}$ due to binary interactions among atoms and protons is mainly based on Refs. [9, 10]. Some modifications, however, are introduced to improve the physical consistency of the model, and analytic fits are presented.

The total excess free energy consists of the reference part $F_{\mathrm{HS}}$, treated in the hard-sphere approximation, and perturbation parts $F_{\text {pert }}$ required to include the attractive (van der Waals) interactions.

\section{Atom-atom interactions}

We treat interactions among neutral species using the fluid perturbation theory of Weeks, Chandler, and Andersen [32] (WCA). An effective potential $\phi(r)$ is separated into the reference and perturbation parts,

$$
\phi(r)=\phi_{\text {ref }}(r)+\phi_{\text {pert }}(r),
$$

where $\phi_{\text {ref }}$ is a purely repulsive finite-range potential which acts at distances $r<r_{*}$, where $r_{*}$ is the minimum point of $\phi(r)$. Accordingly, the free energy splits into the reference and perturbation parts. First we consider the ground state atoms and adopt the interatomic potential 


$$
\phi_{\mathrm{H}}(r)=\phi_{\mathrm{SC}}^{(\mathrm{H})}(r)+\phi_{\mathrm{core}}^{(\mathrm{H})}(r)
$$

where $\phi_{\mathrm{SC}}^{(\mathrm{H})}(r)$ is given by Eq. (2) of Ref. [9], and $\phi_{\text {core }}^{(\mathrm{H})}(r)$ is a correction which acts at short distances $r \lesssim 1.5 \AA$. The correction is required to make $\phi(r)$ go to infinity at $r \rightarrow 0$. Unlike SC 10 who introduced HS cores with fixed ad hoc diameters, we use the scaled mean electric potential of the ground state $\mathrm{H}$ atom:

$$
\phi_{\text {core }}^{(\mathrm{H})}(r)=\frac{e^{2}}{r}\left(1+\frac{r}{a}\right) \mathrm{e}^{-2 r / a} .
$$

This choice seems reasonable since it yields the Coulomb repulsion at $r \rightarrow 0$. We use the effective He screening length [33] $a=\frac{16}{27} a_{\mathrm{B}}$, where $a_{\mathrm{B}}$ is the Bohr radius, for the ground state atom.

The perturbation part of the free energy is

$$
F_{\text {pert }}^{(\mathrm{H})} / N_{\mathrm{H}}=\frac{n_{\mathrm{H}}}{2} \int \phi_{\text {pert }}(r) g_{\text {ref }}(r) \mathrm{d}^{3} r,
$$

where $g_{\text {ref }}(r)$ is the pair correlation function of the reference system. For the HS reference system at low densities, $g_{\text {ref }}(r)$ can be replaced by 0 at $r<d$, and 1 at $r>d$, where $d$ is the HS diameter. Then

$$
F_{\text {pert }}^{(\mathrm{H})} / N_{\mathrm{H}}=-n_{\mathrm{H}} \epsilon_{0}(2 \pi / 3)\left(R_{0}^{3}-d^{3}\right),
$$

where $\epsilon_{0} / k_{\mathrm{B}}=20.2 \mathrm{~K}$ and $R_{0}=8.6 a_{\mathrm{B}}$ for the ground state atom.

The diameter $d$ can be determined from the WCA selfconsistency condition, which involves radial distribution function. However, according to Ref. [34], the WCA value of $d$ at low $\rho$ is close to the Barker's 35 value

$$
d_{\mathrm{B}}=\int_{0}^{\infty}\left[1-\exp \left(-\beta \phi_{\mathrm{ref}}(r)\right)\right] \mathrm{d} r
$$

We adopt $d=d_{\mathrm{B}}$ at $\rho \ll 1 \mathrm{~g} \mathrm{~cm}^{-3}$, and propose the expression $d=d_{\mathrm{B}} \exp \left(-\rho / 2 \mathrm{~g} \mathrm{~cm}^{-3}\right)$ at $\rho \lesssim 1 \mathrm{~g} \mathrm{~cm}^{-3}$. The latter expression fits exact numerical results [9] with an error of about $5 \%$.

We have used the reference part of the potential (A2) in Eq. (A6) and fitted the result by the formula

$$
d_{\mathrm{H}}=d_{0}\left[1+\ln \left(1+c_{1} \sqrt{t}\right)+\frac{t}{1+\ln \left(1+c_{2} t\right)}\right]^{-1}
$$

where $d_{0}=6 a_{\mathrm{B}}, c_{1}=4, c_{2}=0.5$, and $t=T /\left(3.25 \cdot 10^{4} \mathrm{~K}\right)$ for the ground state atom.

We use simple scaling of the potentials for excited states. For two atoms with principal quantum numbers $n$ and $n^{\prime}$, we write

$$
\phi_{n^{\prime} n}(r)=s_{n^{\prime} n}^{-1} \phi_{\mathrm{H}}\left(r / s_{n^{\prime} n}\right)
$$

where $s_{n^{\prime} n}=\left(l_{n}+l_{n^{\prime}}\right) /\left(2 l_{1}\right)$ is the scaling factor, and $l_{n}$ is an average atomic size. The scaling (A\&) ensures the correct Coulomb repulsion at short distances. We estimate $l_{n}^{2}$ as the quantum-mechanical expectation value of $r^{2}$ 36] averaged over the quantum numbers $(l, m)$, which yields

$$
l_{n}=a_{\mathrm{B}} n \sqrt{\left(7 n^{2}+5\right) / 4}
$$

The scaling does not reduce to just multiplying $d_{\mathrm{H}}$ by $s$, but implies simultaneous scaling of the temperature:

$$
d_{n n^{\prime}}(T)=s_{n n^{\prime}} d_{\mathrm{H}}\left(s_{n n^{\prime}} T\right)
$$

The perturbation terms for different states are additive:

$$
F_{\text {pert }}^{(\mathrm{H})}=-\sum_{\kappa \kappa^{\prime}} N_{\kappa} N_{\kappa^{\prime}} a_{\kappa \kappa^{\prime}} / V
$$

where the van der Waals constants $a_{\kappa \kappa^{\prime}}$ are determined by the scaled Eq. (A5),

$$
a_{\kappa \kappa^{\prime}}=-s_{\kappa \kappa^{\prime}}^{2} \epsilon_{0} \frac{2 \pi}{3}\left(R_{0}^{3}-d_{\mathrm{H}}^{3}\left(s_{\kappa \kappa^{\prime}} T\right)\right) \text {. }
$$

\section{Ion-atom interactions}

Following SC [10], we describe the polarization interaction outside the core by the screened dipolar potential:

$$
\phi_{\mathrm{pol}, n}=-\frac{e^{2} \alpha_{n}}{2}\left(\frac{1+r / r_{\mathrm{D}}}{l_{n}^{2}+r^{2}}\right)^{2} \mathrm{e}^{-2 r / r_{\mathrm{D}}},
$$

where $\alpha_{n}$ is the average polarizability of an atom with the principal quantum number $n$, and $r_{\mathrm{D}}$ is the screening length. The rms size $l_{n}$ in the denominator of Eq. (A13) is intermediate between two different values of the polarization radius used in Ref. [10]. Furthermore, the polarizability of $\mathrm{H}$ atom in the state $(\mathrm{nlm})$ averaged over $(\mathrm{lm})$ numbers [36] can be fitted, with an error of $2 \%$, by

$$
\alpha_{n}=0.85 l_{n}^{3}
$$

At short separations, the Coulomb repulsion should prevail. Therefore, in analogy with Eqs. A2, we adopt the interaction potential

$$
\phi_{\text {in }}(r)=\phi_{\text {pol }}(r)+\phi_{\text {core }}^{(\mathrm{in})}(r)
$$

where $\phi_{\text {core }}$ is given by Eq. (A3) with $a=\frac{1}{2} s_{n} a_{\mathrm{B}}$ and the scaling factor $s_{n}=l_{n} / l_{1}$.

The potential (A15) is separated then into the reference and perturbation parts, Eq. (A1), according to the WCA prescription. The perturbation free energy is calculated analogously to Eq. (A4) (with $n_{\mathrm{p}}$ instead of $\frac{1}{2} n_{\mathrm{H}}$ on the right-hand side). This gives

$$
F_{\text {pert }}^{(\text {in })}=-\sum_{\kappa} N_{\mathrm{p}} N_{\kappa} a_{\kappa} / V
$$

where

$$
a_{\kappa}=e^{2} l_{\kappa}^{2}\left[v_{*}\left(l_{\kappa} / r_{\mathrm{D}}\right)-\frac{4 \pi}{3} \phi_{*}\left(l_{\kappa} / r_{\mathrm{D}}\right) d_{*}^{3}\left(s_{\kappa} T\right)\right] .
$$


Here $e^{2} l_{\kappa}^{2} v_{*}$ is the integral of $\phi_{\text {pert }}^{(\mathrm{in})}(r)$ over space, $\left(-e^{2} / l_{\kappa}\right) \phi_{*}$ is the minimum of $\phi_{\text {in }}$, and $d_{\kappa}(T)=$ $l_{\kappa} d_{*}\left(s_{\kappa} T\right)$ is the HS diameter determined by Eq. (A6) (nearly independent of $r_{\mathrm{D}}$ ). We have obtained the fits

$$
v_{*}(x)=\frac{4-1.7 x}{1+1.7 x}, \quad \phi_{*}(x)=\frac{0.155-0.0212 x^{2}}{1+0.34 x^{2}} ;
$$

and $d_{*}(T)$ is given by Eq. (A7) with $d_{0}=0.615, c_{1}=$ $0.71, c_{2}=0.75$, and $t=T /\left(2.15 \cdot 10^{5} \mathrm{~K}\right)$.

\section{Hard sphere contribution}

The HS diameters depend on atomic states and differ for interactions with atoms and ions. Thus we have a non-additive HS mixture. Such mixtures can be described by the van der Waals one-fluid model, which is reasonably accurate for effective filling factors $\eta<0.3$ [37]. In the spirit of this model, we define

$$
\eta=\frac{\pi}{6 N V} \sum_{\kappa} N_{\kappa}\left[\sum_{\kappa^{\prime}} N_{\kappa^{\prime}} d_{\kappa \kappa^{\prime}}^{3}+2 N_{\mathrm{p}} d_{\kappa}^{3}\right]
$$

and use the Carnahan-Starling [38] formula

$$
\beta F_{\mathrm{HS}} / N=\left(4 \eta-3 \eta^{2}\right) /(1-\eta)^{2} .
$$

Finally, the excess free energy associated with the pair potentials is given by Eqs. (A20), (A11) and (A16):

$$
F_{\text {pair }}=F_{\mathrm{HS}}+F_{\text {pert }}^{(\mathrm{H})}+F_{\text {pert }}^{(\mathrm{in})} \text {. }
$$

* E-mail: palex@astro.ioffe.rssi.ru

[1] G. Chabrier and E. Schatzman (editors), The Equation of State in Astrophysics (Cambridge Univ. Press, Cambridge, 1994)

[2] W. Ebeling, W. D. Kraeft, and D. Kremp, Theory of Bound States and Ionization Equilibrium of Plasmas and Solids (Akademie, Berlin, 1977)

[3] C. Alcock and A. F. Illarionov, Astrophys. J., 235, 534 (1980)

[4] M. A. Barstow, J. B. Holberg, and D. Koester, Mon. Not. R. Astron. Soc., 268, L35 (1994)

[5] D. G. Hummer and D. Mihalas, Astrophys. J., 331, 794 (1988)

[6] D. Mihalas, W. Däppen, and D. G. Hummer, Astrophys. J., 331, 815 (1988)

[7] W. Däppen, D. Mihalas, D. G. Hummer, and B. W. Mihalas, Astrophys. J., 332, 261 (1988)

[8] G. Chabrier, J. de Phys. France, 51, 1607 (1990)

[9] D. Saumon and G. Chabrier, Phys. Rev. A, 44, 5122 (1991)
[10] D. Saumon and G. Chabrier, Phys. Rev. A, 46, 2084 (1992)

[11] D. Saumon, G. Chabrier, and H. M. Van Horn, Astrophys. J. Suppl. Ser., 99, 713 (1995)

[12] F. Perrot and C. Dharma-wardana, in The Equation of State in Astrophysics (Ref. 泃), p. 272

[13] H. C. Graboske, D. J. Harwood, and F. J. Rogers, Phys. Rev., 186, 210 (1969)

[14] L. D. Landau and E. M. Lifshitz, Statistical Physics, Pt. I (Pergamon, Oxford, 1986)

[15] S. Ichimaru, Rev. Mod. Phys., 65, 255 (1993)

[16] J. Seidel, S. Arndt, and W. D. Kraeft, Phys. Rev. E, 52, $5387(1995)$

[17] H. E. De Witt, J. Math. Phys., 7, 616 (1966)

[18] J. M. Aparicio and G. Chabrier, Phys. Rev. E, 50, 4948 (1994)

[19] K. Herzfeld, Ann. d. Phys., 51, 261 (1916)

[20] E. Fermi, Zs. f. Phys., 26, 54 (1924)

[21] G. Fontaine, H. C. Graboske, and H. M. Van Horn, Astrophys. J. Suppl. Ser., 35, 293 (1977)

[22] G. Magni and I. Mazzitelli, Astron. Astrophys., 72, 134 (1979)

[23] H. Reinholz, R. Redmer, and S. Nagel, Phys. Rev. E, 52, $5368(1995)$

[24] In Eq. (20) of Ref. [23], the spectroscopic constant 4.75 $\mathrm{eV}$ should be replaced by the dissociation energy $4.48 \mathrm{eV}$, and the factors $\beta h c$ should be inverted (e.g., Ref. [14])

[25] F. J. Rogers, Astrophys. J., 310, 723 (1986)

[26] C. Stehlé and S. Jacquemot, Astron. Astrophys., 271, 348 (1993)

[27] D. R. Inglis and E. Teller, Astrophys. J., 90, 439 (1939)

[28] This difficulty has been pointed out by G. G. Pavlov, Y. A. Shibanov, and V. E. Zavlin (private communication, 1993). It is inevitable, e.g., when the usual Saha equation for the ground state atom yields $n_{\mathrm{p}} / n_{\mathrm{H}}<$ $1-2 \tilde{v}_{11} / \tilde{v}_{\mathrm{p} 1}$. In fact, these authors factorized the last term of Eq. (30) out of the IPF and interpreted it as an interaction term in the free energy instead of a part of $w_{\kappa}$. This, however, did not elminate the problem.

[29] C. A. Iglesias, F. J. Rogers, Astrophys. J., 443, 902 (1995)

[30] F. J. Rogers, F. J. Swenson, and C. A. Iglesias, Astrophys. J., 456, 902 (1996)

[31] L. Collins, I. Kwon, J. Kress, N. Troullier, and D. Lynch, Phys. Rev. E, 52, 6202 (1995)

[32] J. D. Weeks, D. Chandler, and H. C. Andersen, J. Chem. Phys. 54, 5237 (1971); ibid., 55, 5422 (1971)

[33] H. A. Bethe and E. E. Salpeter, Quantum Mechanics of One- and Two-Electron Atoms (Springer, Berlin, 1957)

[34] L. Verlet and J.-J. Weis, Mol. Phys., 24, 1013 (1972); Phys. Rev. A, 5, 939 (1972)

[35] J. A. Barker and D. Henderson, J. Chem. Phys., 47, 2856 (1967); ibid., 47, 4714 (1967)

[36] L. D. Landau and E. M. Lifshits, Quantum Mechanics (Pergamon, Oxford, 1976)

[37] J. Jung, M. S. Jhon, and F. H. Ree, J. Chem. Phys., 100, 528 (1994); ibid., 102, 1349 (1995)

[38] N. F. Carnahan and K. E. Starling, J. Chem. Phys., 51, 635 (1969) 